\title{
An Implementation of Interactive Mobile Terminal Research based on the Android Platform CCar
}

\author{
Feilong $\mathrm{Xu}$ \\ ${ }^{1}$ Institute of Information Technology, Jiangxi University of Technology, Nanchang 330098, China
}

Keywords: Car and interaction; Mobile terminal; Android platform; JAVA language

\begin{abstract}
This paper introduces the overall structure of the hierarchical structure and car Android platform of interactive systems, analyzes the system design and implementation process of each function module. The trial was carried out in the HTCS710e mobile phone and Motorola Xoom tabletcomputer, the test results show that the system has stable operation, friendly man-machine interface, can basicallyachieved our goals at the beginning of design.
\end{abstract}

\section{Introduction}

Computer from birth to now, in the course of development of volume shrinking, while the computational power is constantly increasing, and eventually led to the popular "at the same time, along with the computer, network and mobile communications technology, the development trend of the calculation more freedom and flexibility significantly, network structure and gradually towards pervasive computing model"of traditional distributed system the dispersion of furtherexpanded to a wider geographical area, the use of computing resources but to for multiple users sharing is connected is still not very convenient "appear LAN and work station makes thesharing of resources and use becomes more convenient to move" with the development of wireless networks and the emergence of calculation makes the calculation morefreedom, the user can be any local coverage in wireless communication network architecture! Any time the system resource access popularity, the upcoming computing modewill make the calculation more freedom and flexibility, anyone can at any time! In any place of computing service need, the calculation will be everywhere, diffuse interaction in all places"to serve the needs of the user computing resources throughand the realization of the user interface".

\section{Introduction to the Android platform}

The Android - word means "robot", the name of open-sourcemobile operating system based on Linux platform is also Google company announced on November 5, 2007, is the first mobile terminal to create a truly open and complete system. The platform is composed of an operating system,middleware, user interface and application software component, the structure of the system is shown in Fig. 1.

The platform uses Linux as the core system, at the core of the Linux system, with many control device driver, including Bluetooth (Bluetooth), plug and play USB? Wireless network(Wifi), of course, driver and screen, flash memory and other devices, in the core of Linux system, the use of many of the $\mathrm{C} / / \mathrm{C}++$ language 
developed library (Libraries), including Libc, Open Gl/Es, Web Kit, Sqlite etc.. The Google architecture Java environment Android Runtime, which contains a virtual machine Dalvik Virtual Machine (DVM) and Java function library is the most basic (Core Libraries), Google uses these $\mathrm{C} / \mathrm{C}++$ function library and Java virtual organization builds aset of application framework, to help application developers to develop applications that better (APP). The platform of the Android function library mainly from the following 3 aspects: one is the library comes with the system, the other is a Java of the original, the last one is the open source community library.

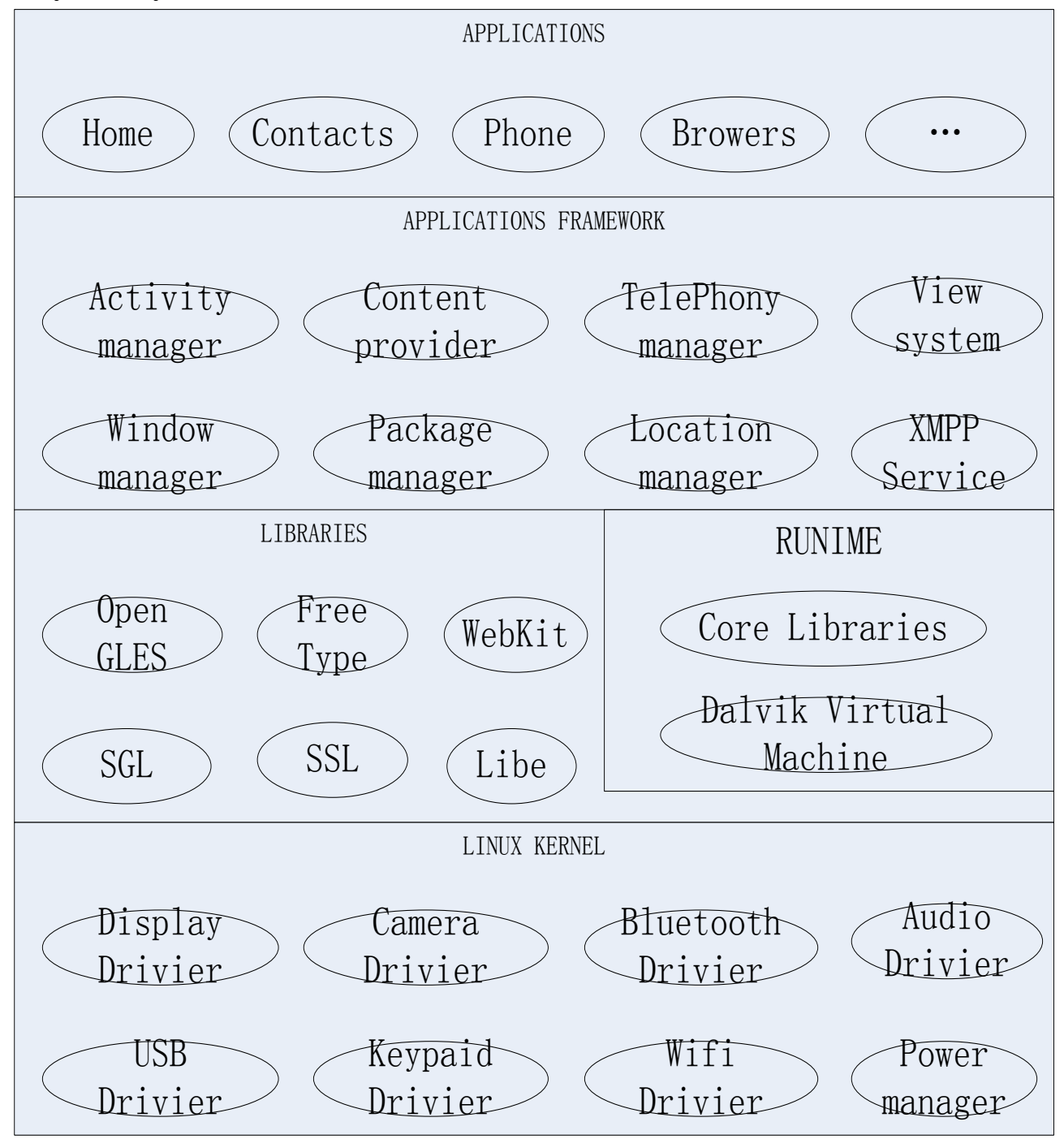

Fig. 1 The hierarchical structure of Android platform

\section{The whole design of the system}

Car and interaction of mobile terminal overall design thinking is the integrated wireless access point of AP in mobile devices on the car (Access Point), as a bridge of communication between intelligent mobile terminal and the driver of the car, intelligent mobile terminal via the Wifi connection and the automobile intelligent mobile device interface, forming a simple wireless local area network.

First of all, in a gesture gesture input interface, then use the software gesture library for matching user input gestures, gestures, finally success, start and gestures corresponding software. 


\section{The design of the system function module}

According to automotive service in all details and the habit of the driver of the car, combined with the principle and technology of gesture, and in full consideration of prototype simulation running effect after the car, will be convenient service terminal design: to gesture definition module, hand gesture starting module, the lock screen module. In addition, to embody the humanized operation, highlight the "people-oriented" this concept, the software also designed a software setting module (including setting color, fast input gesture gesture, shake the mobile phone restart this software, set start gesture sensitivity), especially to the "golden word tower "structure software modules function design is shown in Fig. 2.

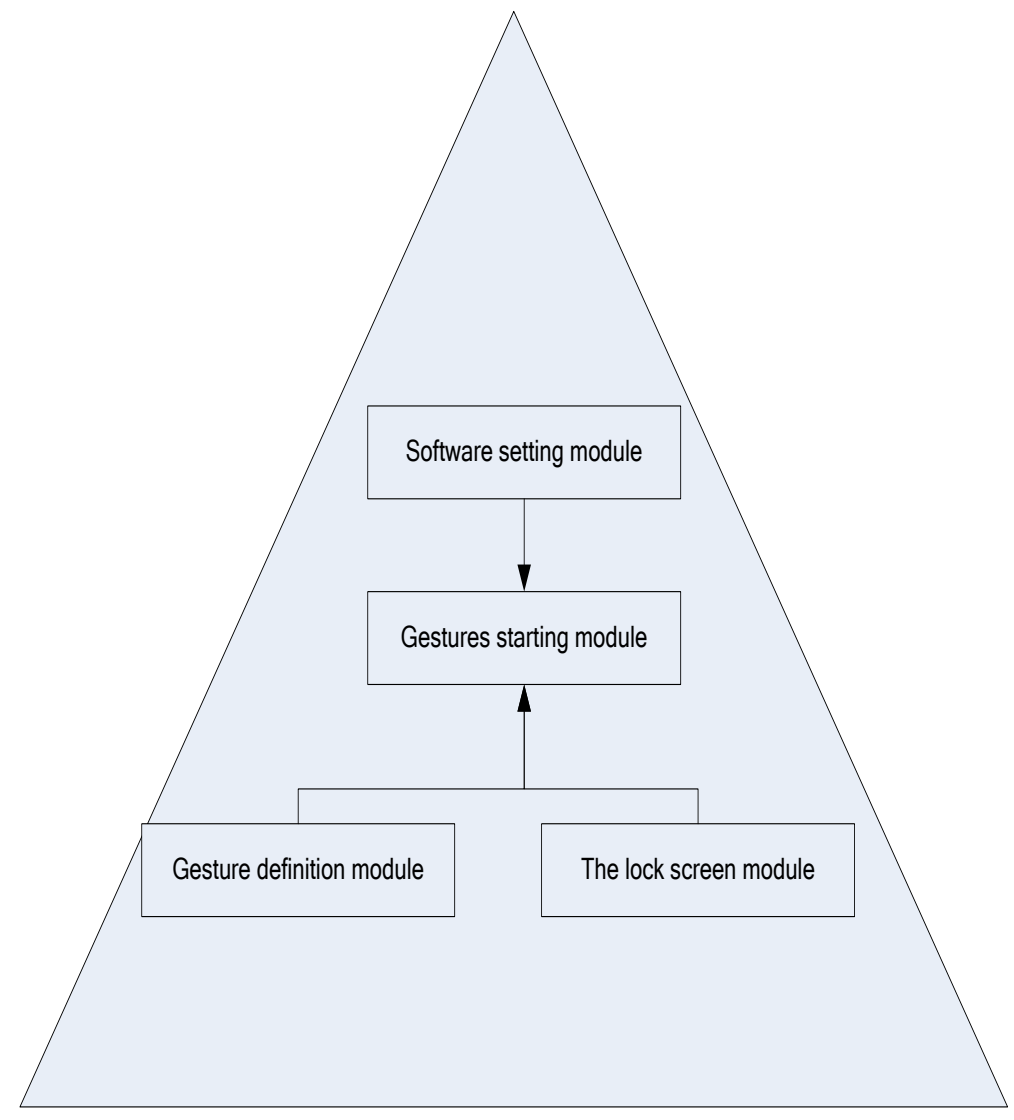

Fig. 2 "pyramid" functional architecture

Gesture definition module is divided into two parts, the gesture list and create gestures which aims to list list, gesture software and user has defined starting gesture, when the user has forgotten, can be found here gesture. Create a gesture and aims to provide a definition of their own personalized start gesture platform for users, allows users to create their own start gesture.

Potentialstarting module is the key module of the whole system, the driver from the gesture input gesture, were matched by gestures starting module and gesture in the list of gestures, finally start and gestures corresponding software.

The lock screen module is one of the highlights of the system, the market nowadays can see Android mobile phone-in mobile phone software gestures start lock screen will enter a state of dormancy, and not a very good complete our needs. The researchers started in the Android mobile phone software gesture based on the original from the new improvement, had now the system. This 
system can make the gesture to start the software lock screen on the phone still run as usual, and not to interfere with the normal use of the lock screen mobile phone.

Purpose of the whole system is "people-oriented", set the software module is to this theme and raise a level, you can set the color, set the startup gesture gesture sensitivity in the module, but also can control whether fast input gestures and shake the phone start this software enabled. Through these settings, a car of my own convenient service terminal building completed

\section{Acknowledgements}

This work was supported by Nature of Jiangxi University of Technology [No. ZR13YB08]

\section{References}

[1] Code Home.Android-An Open Handset Alliance Project. http://code.google.com/android/what-is-an-droid.html . 2008

[2] SHU Xian-hua, DU Zhen-jun, CHEN Rong. Research onMobile Location ServiceDesi gn based on Android. Pro-ceedings of theIEEE Conf WICOM09 . 2009

[3] Tian Yuan, Song Biao, Huh Eui-Nam. Towards the Development ofPersonal Cloud Computing for Mobile Thin-lients. Information Scienceand Applications (ICISA) , 2011 International Conference on . 2011

[4] Na Sang-Ho, Park Jun-Young, Huh Eui-Nam.Personal Cloud Com-puting Security Framework. Services Computing Conference (AP-SCC) , 2010 IEEE Asia-Pacific . 2010

[5] Reto Meier.Professional Android 2 Application Develop-ment. . 2010

[6] Red Hat Bugzilla.Attachment 531725Details for Bug 751297[Z/OL]. https $/ /$ bugzilla.redhat.com/attachment.c gi? id=531725\&action=edit .2012

[7] Ekram Hossain,Garland Chow,Victor C.M. Leung,Robert D. McLeod,Jelena Mi,Vincent W.S. Wong,Oliver Yang. Vehicular telematics over heterogeneous wireless networks: A survey[J]. Computer Communications . 2010 (7)

[8] Chen Wu,Elizabeth Chang. Aligning with the Web: an atom-based architecture for Web services discovery[J]. Service Oriented Computing and Applications . 2007 (2).

[9] A. M. Abbasov,I. M. Aliev,L. H. Kerimova. Optimal location of internet data centers taking into account the risks[J]. Automatic Control and Computer Sciences . 2009 (6)

[10]LuminaryMicro,Inc.LM3S5749Microcontrollerdatasheet. 2009 